\title{
Wavelet Compression and Segmentation of Digital Mammograms
}

\author{
Bradley J. Lucier, Maria Kallergi, Wei Qian, Ronald A. DeVore, Robert A. Clark, Edward B. Saff, \\ and Laurence P. Clarke
}

\begin{abstract}
An initial evaluation of Haar wavelets is presented in this study for the compression of mammographic images. Fifteen mammograms with $105 \mu \mathrm{m}$ / pixel resolution and varying dynamic range (10 and 12 bits per pixel) containing clustered microcalcifications were compressed with two different rates. The quality and content of the compressed reconstructed images was evaluated by an expert mammographer. The visualization of the cluster was on the average good but degraded with increasing compression because of the discontinuities introduced by these types of wavelets as the compression rate increases. However, the artifacts in the decoded images were seen as totally artificial and were not misinterpreted by the radiologist as calcifications. The classification of the parenchy. mal densities did not change significantly but the morphology of the calcifications was increasingly distorted as the compression rate increased leading to lower estimates of the suspiciousness of the cluster and higher uncertainties in the diagnosis. The uncompressed and two sets of compressed images were also processed by a wavelet method to extract the calcifications. Despite the fact that the segmentation algorithm generated several false-positive signals in highly compressed images, all true clusters were successfully segmented indicating that the compression process preserved the features of interest. Our preliminary results indicated that wavelets could be used to achieve high compression rates of mammographic images without losing small details such as microcalcification clusters as well as detect the calcifications from either the uncompressed or compressed reconstructed data. Further research and application of multiresolution analysis to digital mammography is continuing.

Copyright 1994 by W.B. Saunders Company
\end{abstract}

KEY WORDS: wavelets, image compression, segmentation, digital mammography.

D IGITAL MAMMOGRAPHY involves either the digitization of screen-film mammograms or the direct digital acquisition of $\mathrm{x}$-rays. In both cases, the requirements for high resolution images with high dynamic range lead to large data sets, on the average about 50 Mpixels with dynamic ranges of 12 bits per pixel. ${ }^{1,2}$ In addition, screening digital mammography implies real time availability to the radiologist of a series of such images per patient for comparative study and accurate diagnosis. ${ }^{3}$ Such large image database challenges the existing technology for data storage, transmission, and display. Data compression methods could facilitate these processes but they should also satisfy the requirements for highly accurate reconstruction of mammograms.

Several lossless and lossy compression methods have been investigated for medical imaging applications. ${ }^{4,5}$ Lossless compression methods applied to mammography include tree-based codes which represent a large class of variablelength encoding schemes and arithmetic codes. ${ }^{6}$ Lossless methods have the advantage that they can be applied anywhere because such compressed images are reconstructed without error. Their disadvantage is the small compression rates, on the order of $3: 1$. In contrast, lossy techniques can achieve very high compression ratios at the expense of errors in the reconstructed images. A new class of lossy compression algorithms is currently under development that could offer data with no visible artifacts. ${ }^{7,8}$ Receiver operating characteristic (ROC) analysis on lossy compression showed that it is possible to use lossy techniques in medical imaging, provided that the diagnostic power is not lost or diminished..$^{9,10}$ Such methods are referred to as "visually lossless" and hold promise for effective mammogram compression. The properties of the human visual system are such that some losses can be tolerated without affecting the visual evaluation of an image which, despite the losses, appears identical to the original. Furthermore, even visually lossy im-

From the Department of Mathematics, Purdue University, W. Lafayette, IN, Departments of Radiology and Mathematics, University of South Florida, Tampa, FL, and the Department of Mathematics and Statistics, University of South Carolina, Columbia, SC.

Supported in part by the Office of Naval Research (Contracts N00014-91-J-1152 and N00014-91-J-1076), the Army High Performance Computing Research Center at the University of Minnesota, and the H. Lee Moffitt Cancer Center \& Research Institute at the University of South Florida.

Address reprint requests to Laurence P. Clarke, PhD, Department of Radiology, University of South Florida, 12901 Bruce B. Downs Blvd, Box 17, Tampa, FL 33612-4799.

Copyright $\odot 1994$ by W.B. Saunders Company

0897-1889/94/0701-0005\$03.00/0 
ages may be acceptable, when artifacts due to lossy compression can be recognized as entirely artificial and do not disturb the discriminating analysis followed by the radiologist to reach a diagnosis. Finally, it should be pointed out that the use of lossy compression methods depends on the application, the image, and the aims of a particular project. 5

One of the most promising lossy compression approaches uses wavelets. ${ }^{11}$ Wavelets have been already used for mammogram segmentation, ${ }^{12,13}$ enhancement, ${ }^{12}$ and compression. ${ }^{7}$ The present study focuses on the use of Haar wavelets for the compression of mammographic images containing clustered calcifications. Uncompressed and compressed reconstructed data were also processed with a segmentation algorithm that used a wavelet transform. A radiologist expert in mammography evaluated the quality of the compressed images and the usefulness of the segmented data based on a questionnaire designed to provide a qualitative description of the original and compressed images. An extensive ROC clinical evaluation is beyond the scope of this research that aims primarily at preclinical evaluation and algorithm optimization. The purpose of the study is three-fold: (1) to apply different levels of compression to digital mammograms and determine the limits of acceptable losses, which will serve as a first criterion to the selection of an optimum wavelet compression approach, (2) to evaluate the quality of the lossy compressed images and the effect of the losses in the radiologist's diagnosis, and (3) to show the feasibility of extracting the calcifications from the uncompressed and compressed data with a wavelet-based segmentation algorithm, a process that could provide an additional criterion to the design of an optimum compression method for digital mammography.

\section{MATERIALS AND METHODS}

\section{Mammograms}

Fifteen screen-film mammograms were considered as a first test of the compression algorithm. They all contained one biopsy-proven malignant cluster of calcifications superimposed on parenchymal tissues of varying density. All mammograms were digitized at a resolution of $105 \mu \mathrm{m} /$ pixel with a DuPont NDT Scan II digitizer (Delaware, VA). Seven images had a dynamic range of 10 bits per pixel $(1,024$ gray levels) and eight had a dynamic range of 12 bits per pixel $(4,096$ gray levels). The optical density range selected for the digitization was different for each mammogram and it was determined from the optical density of the brightest spot on the films. This allowed the maximization of the intensity differences between the various breast features and thus maximum separation in pixel values between the microcalcifications and the surrounding tissues. Sections of the images $(512 \times 512$ pixels $)$ containing the calcification cluster were used for compression and segmentation. The sizes of the original images were 327,680 and 393,216 bytes for the 10-bit and 12-bit files, respectively.

\section{Image Compression}

A mathematical theory that analyzes the efficiency of wavelet-based image compression schemes is described in detail elsewhere. ${ }^{11}$ The method used in this study for the compression of mammographic images is identical to that used to report the results of Fig 14 in DeVore ${ }^{11}$ and only a brief description of the method will be presented here.

A wavelet-based compression algorithm is constituted of three steps: ${ }^{11}$ (1) A wavelet family is selected that will be used to decompose the image. Each wavelet family has different smoothness characteristics and approximation properties; in this article, the Haar wavelets are chosen based on our previous experience. ${ }^{11}$ (2) A quantization strategy must be selected. Each strategy is equivalent to a metric with which the difference between the original and reconstructed images will be measured. Two popular choices of metric are the $\mathrm{L}^{2}$ (mean-square error) and $\mathrm{L}^{1}$ (mean-absolute error) metrics. If the pixels of the original image $f$ are denoted by $p_{j}, j=\left(j_{1}, j_{2}\right)$ and $1 \leq j_{1}, j_{2} \leq 512$, and the pixels of the reconstructed image $\tilde{f}$ are denoted by $\tilde{p}_{j}$, then the mean-square error is defined by

$$
\|f-\tilde{f}\|_{2}:=\left(\frac{1}{N} \sum_{j}\left|p_{j}-\tilde{p}_{j}\right|^{2}\right)^{\frac{1}{2}}
$$

and the mean-absolute error is defined by

$$
\|f-\tilde{f}\|_{1}:=\frac{1}{N} \sum_{j}\left|p_{j}-\bar{p}_{j}\right|,
$$

where $N$ is the number of pixels in the image; in 
this study $\mathrm{N}=512 \times 512=262,144$. The quantization strategy determines the relative importance of contrast and spatial frequency in choosing which features of the image can be removed while causing minimal visual degradation. It was previously reported ${ }^{11}$ that for $512 \times$ 512 natural images viewed at a standard viewing distance of four times the width of the image, the contrast-frequency tradeoff implied by the $\mathrm{L}^{1}$ error metric more closely matched the characteristics of the human visual system than the contrast-frequency tradeoff implied by the choice of the $\mathrm{L}^{2}$ error metric. Mammograms are not viewed by radiologists solely at arm's length, however, and it was observed with earlier 8-bit digitized mammograms that the $\mathrm{L}^{2}$ metric balanced the degradation of the edges and shapes of microcalcification clusters with the degradation of the structure of architectural distortions in the underlying tissues. The $\mathrm{L}^{1}$ quantization strategy at a given level of compression tended to preserve the structure of architectural distortions better than the $\mathrm{L}^{2}$ strategy, at the expense of smoothing away evidence of microcalcifications. Therefore, for our first controlled study, we chose to use only the $\mathrm{L}^{2}$ quantization strategy. The results of this work will serve as guidelines for our future investigations of other quantization strategies.

(3) After choosing the wavelets and quantization strategy, one must select a parameter called the maximum quantization interval, $q$, which roughly determines the local error. The greater the $q$ is, the greater the compression, but also the greater the error. When dealing with images with different dynamic ranges (in this study, for example, seven of the images have 1,024 grey levels and eight have 4,096 grey levels), it is useful to consider the normalization of the intensity range to a minimum of 0 and a maximum of 1 . This can be done by dividing all pixel values by the maximum grey scale value $2^{\mathrm{K}}$, where $K=10$ or 12 in this study. Then, the parameter that best estimates the local visual quality of the image is $q / 2^{\mathrm{K}}$. Thus, $q=512$ for our 12-bit images leads to comparable compression ratios and local image quality as $q=128$ for our 10-bit images. When comparing meansquare errors, it is again useful to divide by the maximum grey scale. Thus, a mean-square error of 16 in a 10-bit image is roughly comparable with a mean-square error of 64 in a 12-bit image (or of 4 in an 8-bit image). Based on this reasoning, 15 mammograms were compressed twice with the parameters summarized in Table 1.

The compression algorithm was implemented in FORTRAN and C-language and run on a Sun Microsystems (Mountain View, CA) SPARCstation 2. The time of compression was 10 seconds per image.

\section{Image Segmentation}

A wavelet-based segmentation method has been previously applied to uncompressed digital mammograms for the extraction of calcification clusters. ${ }^{12}$ Those results were very promising with $100 \%$ sensitivity and only .1 falsepositive clusters per image. The performance of this algorithm on the current uncompressed and compressed data could, therefore, be a first indication of the effect of wavelet compression on the design of computer assisted diagnosis methods or compensate for the losses allowing higher compression rates. Based on this hypothesis, the images of this study were processed as follows: (1) They were enhanced with a treestructured nonlinear filter. As mentioned in detail elsewhere, this filter consists of a series of central weighted median filters which are applied to the image in three steps. This process allows effective suppression of the image noise without removing image detail. ${ }^{12,14}$ (2) The enhanced image was decomposed using a twochannel wavelet transform into four independent subimages which contain different types of frequencies. ${ }^{12,15,16}$ The first subimage contains the low frequencies of the image, the second contains the vertical high and horizontal low frequencies, the third contains the vertical low and horizontal high frequencies, and the fourth contains the high frequencies in both directions. (3) A wavelet reconstruction process was performed using the last three subimages resulting in one image that contains features correspond-

\begin{tabular}{ccc}
\multicolumn{3}{c}{ Table 1. Parameters Used for Two Compression Runs of 15} \\
Mammograms \\
\hline Parameter & Set No 1 & Set No 2 \\
\hline$q$ & $128-10$-bit images & 256-10-bit images \\
& $512-12$-bit images & 1024-12-bit images \\
Space $L^{p}$ & $\mathrm{p}=2$ & $\mathrm{p}=2$ \\
\hline
\end{tabular}

q, maximum quantization interval. 
ing to calcifications. Details of this algorithm are described elsewhere ${ }^{12,15,16}$ but it should be mentioned that the proposed wavelet segmentation could be further improved and optimized for compressed images.

Once the parameters of the segmentation algorithm are optimized for the uncompressed mammograms, they are kept constant for both uncompressed and compressed images. The software was written in C-language and run on a Sun Microsystems (Mountain View, CA) SPARCstation 2. The time of segmentation was about 5 minutes per image.

\section{Evaluation Protocol}

A questionnaire was formed for the qualitative visual analysis of the original, compressed, reconstructed, and segmented versions of the 15 cases. It included nine questions shown in Table 2. Each question was rated on a scale of 1 to 5 . There were 7 images to be evaluated per case, namely the film, the digitized image, the reconstructed image from the first compression, the reconstructed image from the second compression, and three segmentation results. A radiologist expert in mammography was asked to read all 105 images and complete one questionnaire for each one although some of the questions were not applicable to all of them. The objective of this evaluation was to obtain a standard description of the visual characteristics of the quality of the digitized and compressed reconstructed images, to determine the degree of assistance of the segmentation algorithm to the recognition of the calcification clusters, and to have a first estimate of the acceptable limits for the lossy compression of mammograms.

Digital images were displayed on a $19^{\prime \prime}$ color Sun monitor with $1,152 \times 900$ pixels using SunVision 1.2 software; no processing of the displayed images was permitted. It should be noted that current technology limits the display to 8 bits per pixel or 256 grey levels. With SunVision 1.2, the lowest values in the 10-bit or 12-bit images are scaled to 0 , the highest values to 255 , and the intervening values are scaled proportionately between 0 and 255; lower and higher values are clamped to 0 and 255 , respectively.

\section{RESULTS AND DISCUSSION}

Figures 1 and 2 present sections of digitized mammograms ( $105 \mu \mathrm{m}, 12$ bits per pixel) before and after compression. The mammograms contain one cluster of biopsy-proven, malignant calcifications, the location of which is determined by an expert mammographer and is indicated by an arrow. There is a variation in the subtlety of the calcifications caused mainly by the density of the surrounding or superim-

Table 2. Questionnaire Used for Qualitative Evaluation of Compressed Images and Segmentation Data

\begin{tabular}{|c|c|c|c|c|c|}
\hline Characteristic & & & Rating Scale & & \\
\hline \multirow[t]{2}{*}{ 1. Visualization of calcification cluster } & 1 & 2 & 3 & 4 & 5 \\
\hline & Poor & & & & Excellent \\
\hline 2. Number of calcifications & 1.5 & $6-10$ & $11-20$ & $21-30$ & $>30$ \\
\hline \multirow[t]{2}{*}{ 3. Parenchymal density classification } & 1 & 2 & 3 & 4 & 5 \\
\hline & Low & & & & High \\
\hline \multirow[t]{3}{*}{ 4. Degree of suspicion } & 1 & 2 & 3 & 4 & 5 \\
\hline & Definitely & & Undefined & & Definitely \\
\hline & Normal & & & & Cancer \\
\hline \multirow[t]{2}{*}{ 5. Distortion of calcifications' morphology } & 1 & 2 & 3 & 4 & 5 \\
\hline & Extreme & & & & None \\
\hline \multirow[t]{2}{*}{ 6. Distortion of parenchyma } & 1 & 2 & 3 & 4 & 5 \\
\hline & Extreme & & & & None \\
\hline \multirow[t]{2}{*}{ 7. Degree of losses (overall) } & 1 & 2 & 3 & 4 & 5 \\
\hline & Extreme & & & & None \\
\hline \multirow{3}{*}{$\begin{array}{l}\text { 8. Degree of confusion secondary to false } \\
\text { positive artifacts }\end{array}$} & & & & & \\
\hline & 1 & 2 & 3 & 4 & 5 \\
\hline & Extreme & & & & None \\
\hline \multirow{2}{*}{$\begin{array}{l}\text { 9. Degree of assistance of segmentation } \\
\text { process }\end{array}$} & 1 & 2 & 3 & 4 & 5 \\
\hline & Extreme & & & & None \\
\hline
\end{tabular}

One answer sheet was used for each image, namely the original film, the digitized mammogram, the compression number 1, and the compression number 2 , although some of the questions were not applicable to all images. 


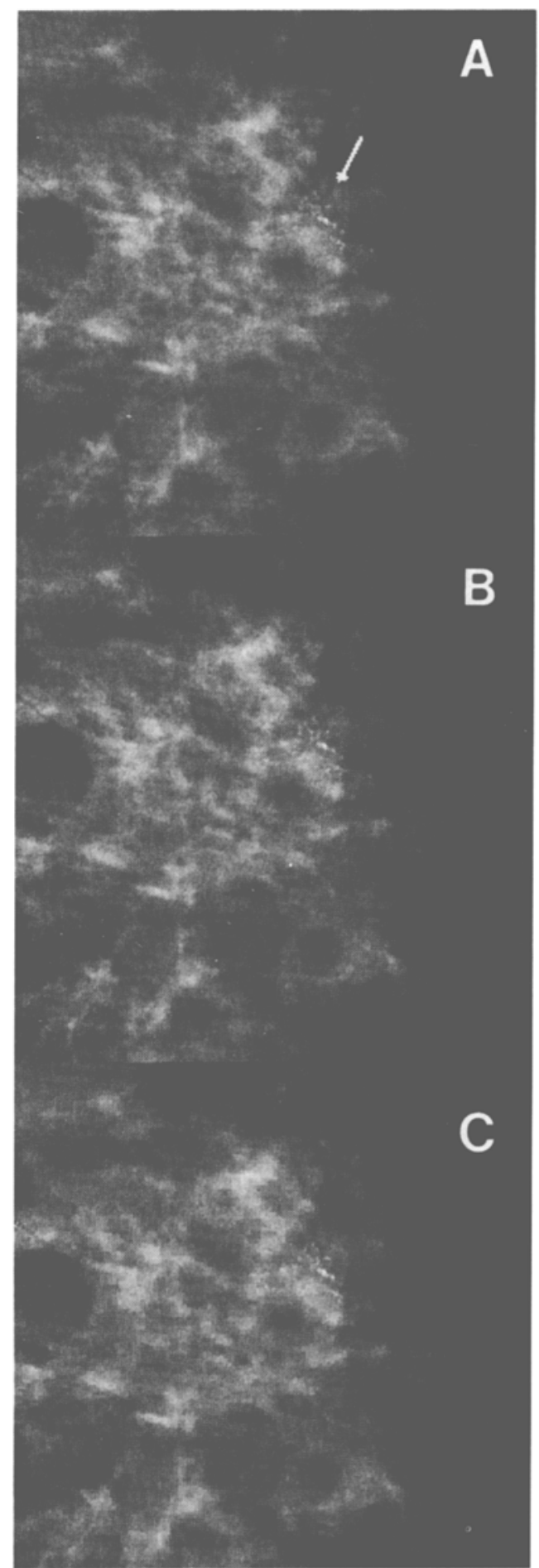

Fig 1. (A) Section of digitized mammogram ( $105 \mu \mathrm{m}$, 12 bits per pixel] with a calcification cluster of average subtlety indicated by arrow. (B) Wavelet compressed and recon" structed image with maximum quantization interval $q=512$; mean-square error, 37.14; and compression ratio 53:1. (C) Wavelet compressed and reconstructed image with $q=1,024$ : mean-square error, 48.99; and compression ratio 123:1.

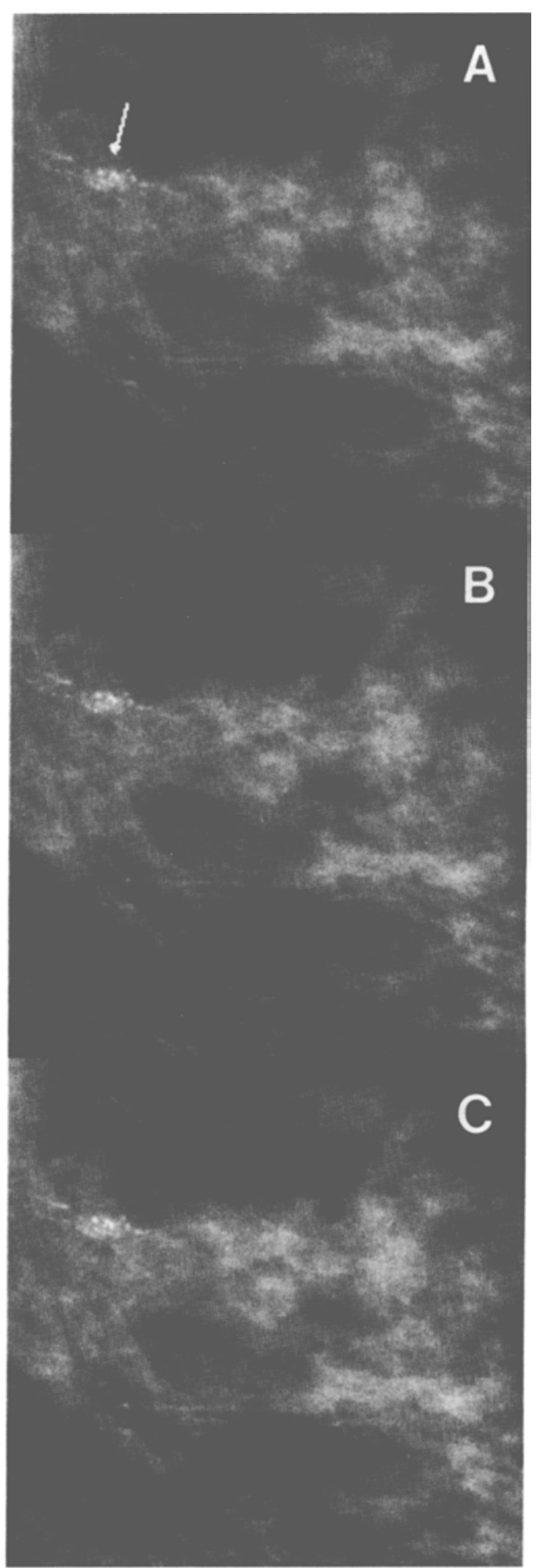

Fig 2. (A) Section of digitized mammogram ( $105 \mu \mathrm{m}, 12$ bits per pixel) with a more subtle calcification cluster indicated by arrow. (B) Wavelet compressed and reconstructed image with maximum quantization interval $q=512$; mean-square error, 54.61; and compression ratio 22:1. (C) Wavelet compressed and reconstructed image with $q=1,024$; mean-square error. 74.51; and compression ratio 55:1. 
posed parenchymal tissues. The mammogram of Fig $1 \mathrm{~A}$ has a parenchymal density of about $50 \%$ and the mammogram of Fig $2 \mathrm{~A}$ has a density of about $75 \%$ of the total breast area. The compressed reconstructed images with a maximum quantization interval of 512 are shown in Figs $1 \mathrm{~B}$ and $2 \mathrm{~B}$. Figures $1 \mathrm{C}$ and $2 \mathrm{C}$ show the corresponding compressed reconstructed images using a maximum quantization interval of 1,024. Because of the differences in the parenchymal density and the noise content, different compression ratios and different mean-square errors were obtained for the same $q$ value.

The results of the compression can be seen better in Figs 3 and 4 where horizontal crosssections through the calcification cluster of the images in Figs 1 and 2, respectively, are plotted. The continuity and smoothness of the pixels in the original images is increasingly lost as the maximum quantization interval and thus the compression rate increases. The higher compression ratio observed for the first mammogram is due to its lower parenchymal density which also accounts for the fact that the calcifications are better preserved in this case even at the higher compression $(q=1,024)$. On the other hand, the data are smoother in the second mammogram where smaller compression rates are obtained. In general, the compression effects are different for mammograms of low and high parenchymal densities. Furthermore, mammograms digitized at higher dynamic range (more gray scales) allow higher compressions with less information loss.

Table 3 lists the mean-square errors and the compression ratios achieved in the two compression sets. The compression ratios were determined by the number of bytes of the original files divided by the corresponding compressed sizes. It is best to interpret the mean-square errors by dividing by the number of gray scales in each image. Therefore, for the first compression results of Fig 1B, the mean-square error is $37.14 / 4,096$ or $9 \%$ whereas for the second compression of Fig $1 \mathrm{C}$, it is $48.99 / 4,096$ or $1.2 \%$. Similarly, the mean-square errors of the images in Figs $2 B$ and $2 C$ are $54.6 / 4,096$ or $1.3 \%$ and $74.51 / 4,096$ or $1.8 \%$, respectively.

Figures 5 and 6 show the segmentation results for the images in Figs 1 and 2. The algorithm
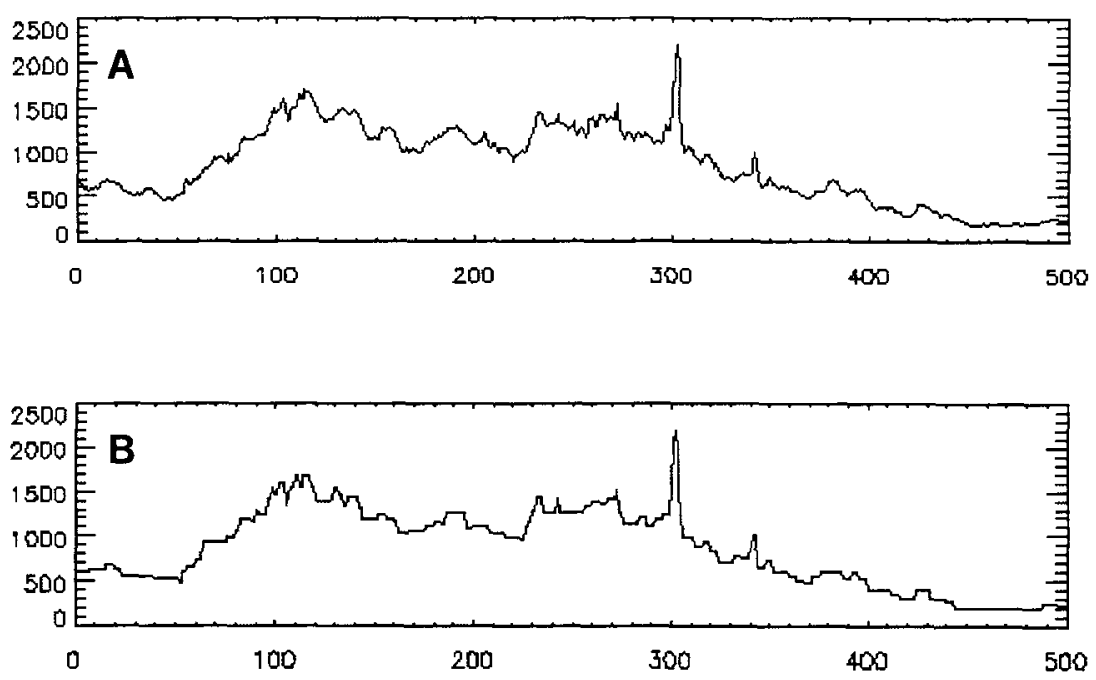

Fig 3. Horizontal cross-sections through the calcification cluster shown in Fig 1, A through C. (A) Original uncompressed data, (B) compressed data with $q=512$, and $(C)$ compressed data with $q=1,024$. 


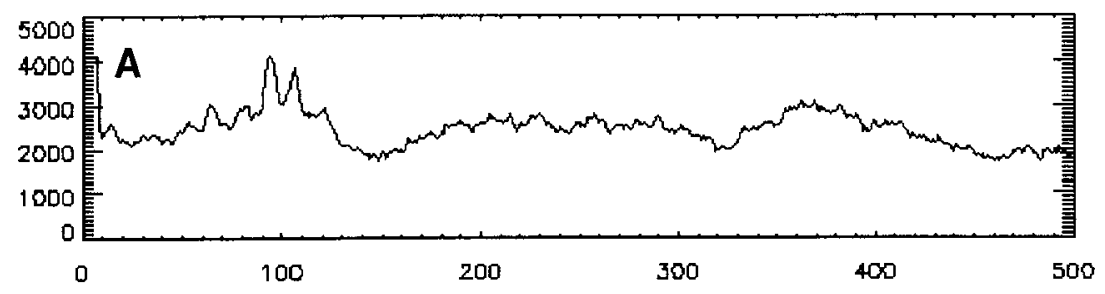

Fig 4. Horizontal cross-sections through the calcification cluster shown in Fig 3, A through C. (A) Original uncompressed data, (B) compressed data with $q=512$, and $(C)$ compressed data with $q=1,024$.
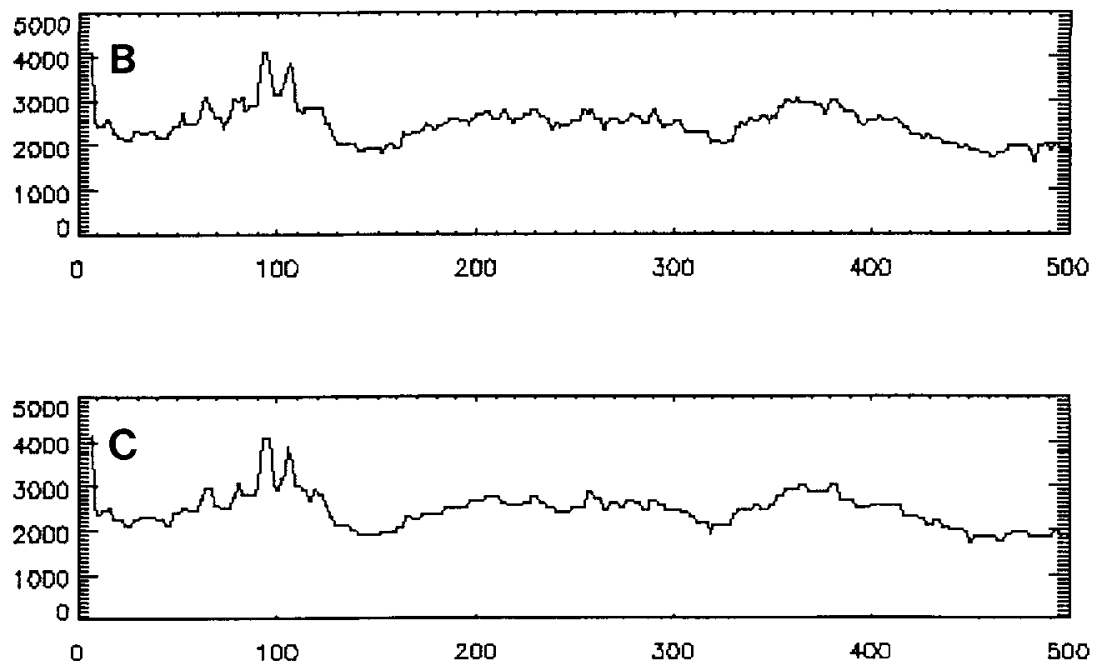

was successful in segmenting the calcifications from the uncompressed images. The loss of continuity and smoothness of the pixel in the compressed data affects the results of the segmentation algorithm in that it generates falsepositive spots which, although not of the same intensity as those corresponding to the calcifica- tions, are considered as distracting by the radiologist, particularly for the higher compression (set number 2). This indicates that either a different segmentation algorithm should be developed for compressed data or a different compression method should be used to avoid abrupt discontinuities in the compressed im-

Table 3. Dynamic Ranges, Mean-Square Errors Measured in Gray Scales and Compression Ratios for the Two Compression Sets That Have Different Maximum Quantization Intervals

\begin{tabular}{rccccc}
\hline $\begin{array}{c}\text { Image } \\
\text { No }\end{array}$ & Gray Levels & $\begin{array}{c}\text { Mean-Square } \\
\text { Error (set no 1) }\end{array}$ & $\begin{array}{c}\text { Compression } \\
\text { Ratio (set no 1) }\end{array}$ & $\begin{array}{c}\text { Mean-Square } \\
\text { Error (set no 2) }\end{array}$ & $\begin{array}{c}\text { Compression } \\
\text { Ratio (set no 2) }\end{array}$ \\
\hline 1 & 4,096 & 36.02 & 69 & 45.46 & 158 \\
2 & 4,096 & 37.14 & 53 & 48.99 & 123 \\
3 & 4,096 & 48.06 & 30 & 64.42 & 74 \\
4 & 4,096 & 52.51 & 25 & 70.61 & 68 \\
5 & 4,096 & 55.00 & 24 & 73.32 & 66 \\
6 & 4,096 & 29.47 & 77 & 39.09 & 54.94 \\
7 & 4,096 & 45.28 & 25 & 74.51 & 56 \\
8 & 4,096 & 54.61 & 22 & 16.03 & 113 \\
9 & 1,024 & 13.54 & 29 & 16.34 & 126 \\
10 & 1,024 & 13.94 & 29 & 12.40 & 182 \\
11 & 1,024 & 10.73 & 36 & 15.31 & 152 \\
13 & 1,024 & 13.24 & 34 & 15.28 & 156 \\
14 & 1,024 & 13.32 & 37 & 15.88 & 138 \\
\end{tabular}




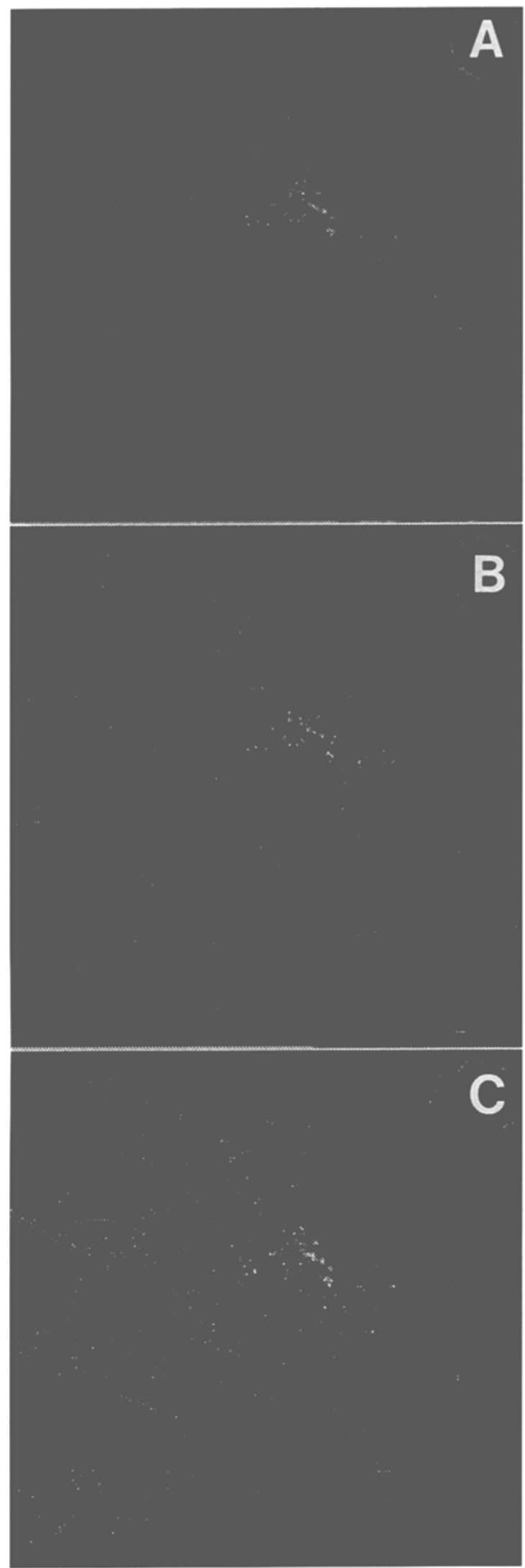

Fig 5. Segmentation results of the images in Fig 1 using two-channel wavelet decomposition and reconstruction. Segmented calcifications from (A) original digitized image, (B) compressed reconstructed data with $q=512$, and $(C)$ compressed reconstructed data with $q=1,024$.

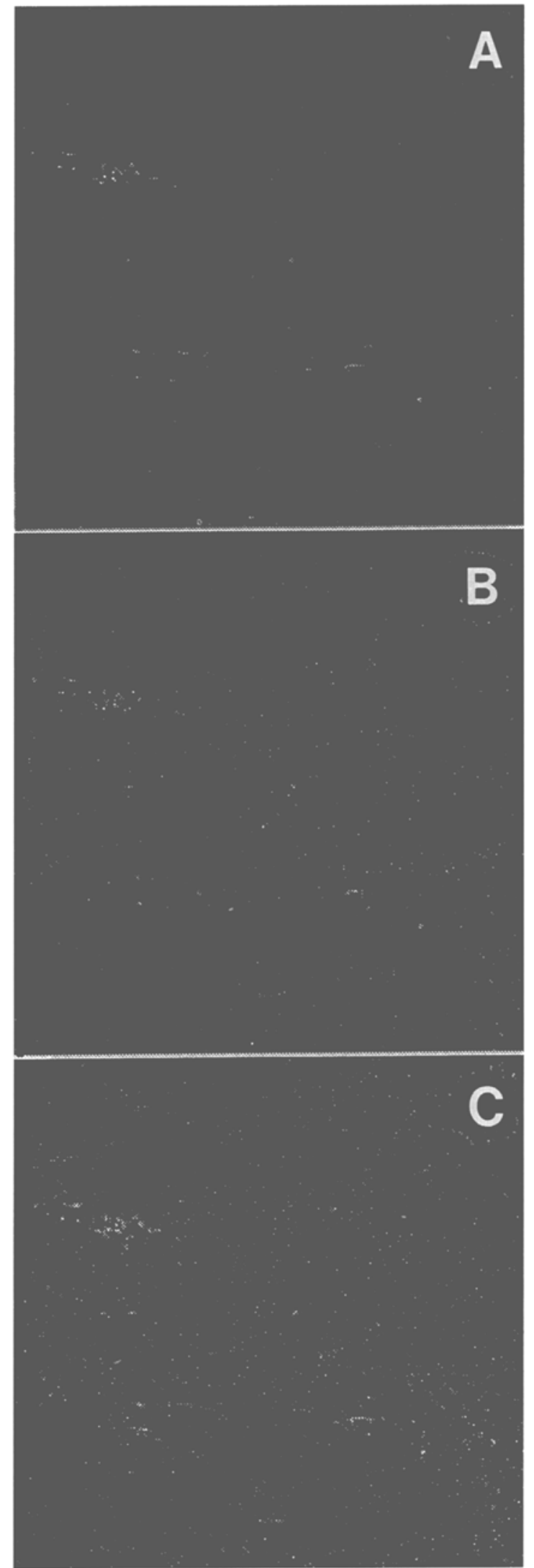

Fig 6. Segmentation results of the images in Fig 2 using two-channel wavelet decomposition and reconstruction. Segmented calcifications from (A) original digitized image, (B) compressed reconstructed data with $q=512$, and $(C)$ compressed reconstructed data with $q=1,024$. 
ages. A solution to the Iatter can also be a different family of wavelets, currently under investigation, which would result in smoother images with smaller gradient.

It should be pointed out that the Haar wavelets used in this study are inherently nonsmooth compression techniques and are expected to be discontinuous across lines that run vertically and horizontally. Thus, at very high compression ratios, the artifacts introduced into the compressed images consist of straight lines and corners where these lines intersect. Using smoother wavelets would introduce different artifacts. The Haar wavelets, however, have the advantages that (1) they do not remove any significant information pertaining to the calcification clusters as indicated by the unstructured difference images between the original and compressed reconstructed data and (2) they introduce artifacts that can be seen by the radiologist as totally artificial; indeed, there were no false-positive readings of microcalcifications by the radiologist on the compressed images. In contrast, the wavelet segmentation algorithm misread many of the edge artifacts as false-positive signals, which made some of the compressed segmented images less useful to the radiologist as a tool for diagnosis. It remains to be seen whether the advantages of smoother wavelets (no edges in the reconstructed images, fewer false-positive detections by the segmentation algorithm) will outweigh the disadvantages (introducing artifacts that may be misread by the radiologist as microcalcifications or other structures).

The results of the evaluation of the visualization of the clustered calcifications are shown in Fig 7 for the film, the uncompressed and the two sets of compressed data. In $60 \%$ of the films, the visualization of the cluster is classified as excellent; in $13 \%$ the visualization of the clusters is very good, and in $27 \%$ is good (Fig 7A). The same $60 \%$ and $13 \%$ of excellent and very good cluster visualization respectively was obtained from the digitized uncompressed images (Fig 7B). However, only $8 \%$ were classified as good while another $7 \%$ was classified as mediocre. The plots in Figs $7 \mathrm{C}$ and $7 \mathrm{D}$ show that the first
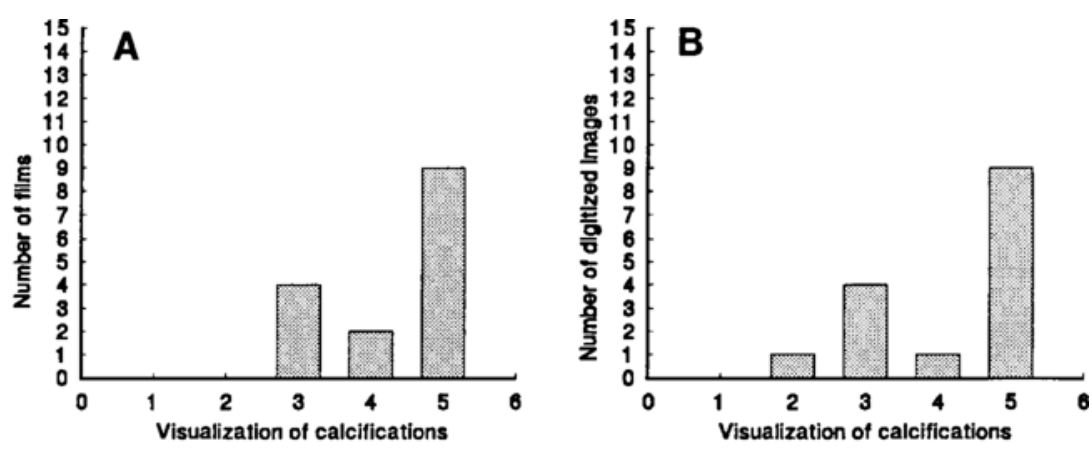

Fig 7. Bar diagrams of the classification of the visualization of the calcifications in (A) the 15 films, $(B)$ the uncompressed digitized images, (C) the first set of compressed images, and (d) the second set of compressed images. The scale is 1 , poor; 2 , mediocre; 3 , good; 4 , very good, 5 , excellent.
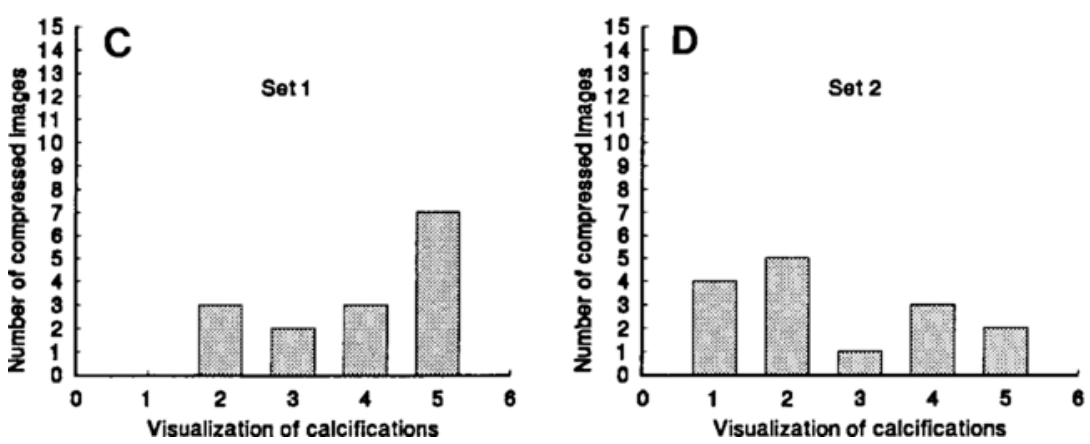
compression run with $q=128$ or 512 demonstrates $47 \%$ excellent visualization for compressions up to $53: 1,20 \%$ very good, $13 \%$ good, and $20 \%$ mediocre. The visualization degraded during the second compression run where the following percentages were calculated from the completed answer sheets: $13 \%$ excellent for compressions up to $56: 1,20 \%$ very good, $7 \%$ good, $33 \%$ mediocre, and $27 \%$ poor. It should be noted that the quality of the compressed data depends on the image contents, namely the parenchymal density and the image noise.

The radiologist's estimates of the losses after compression led to the bar diagrams of Fig 8 . Losses included artifacts not present in the films, changes in the morphology of the cluster and the individual calcifications, and distortion of the breast parenchyma. Assuming no losses for the films, the losses in the digitized mammograms and the compressed images are graded on a scale of 1-5 from extreme to none. Scale number 3 corresponded to average losses and was set as the limit of the visually acceptable level of losses for the purpose of this study. Figure $8 \mathrm{~B}$ shows that only $13 \%$ of the first set of compressed images exceeded the average losses (grade number 3 ). Furthermore, the major contribution to this percentage was from the 10-bit images. In contrast, $73 \%$ of the higher compression results exceeded the average losses.

The analysis of the remainder of the expert's evaluation indicates the following: (1) In $90 \%$ of the cases, the number of calcifications estimated within a cluster is the same between the film, the digitized mammogram, and the first compression set and falls one range lower for the second compression set. (2) In $67 \%$ of the cases, the parenchyma density estimation and classification remains the same between the film, the digitized images, and the first compression set while a higher estimate is given for the second compression set. (3) In $33 \%$ of the cases, the same degree of suspiciousness of the lesion was given from the film, the digital mammogram and the compressed images. However, in the remaining $67 \%$, the compressed data gave the impression of a less suspicious case than the originals. (4) The morphology of the calcifications (shape, size, and geometry) degrades in the entire second set of compression by one or two levels. Fifty-three percent of the first com-
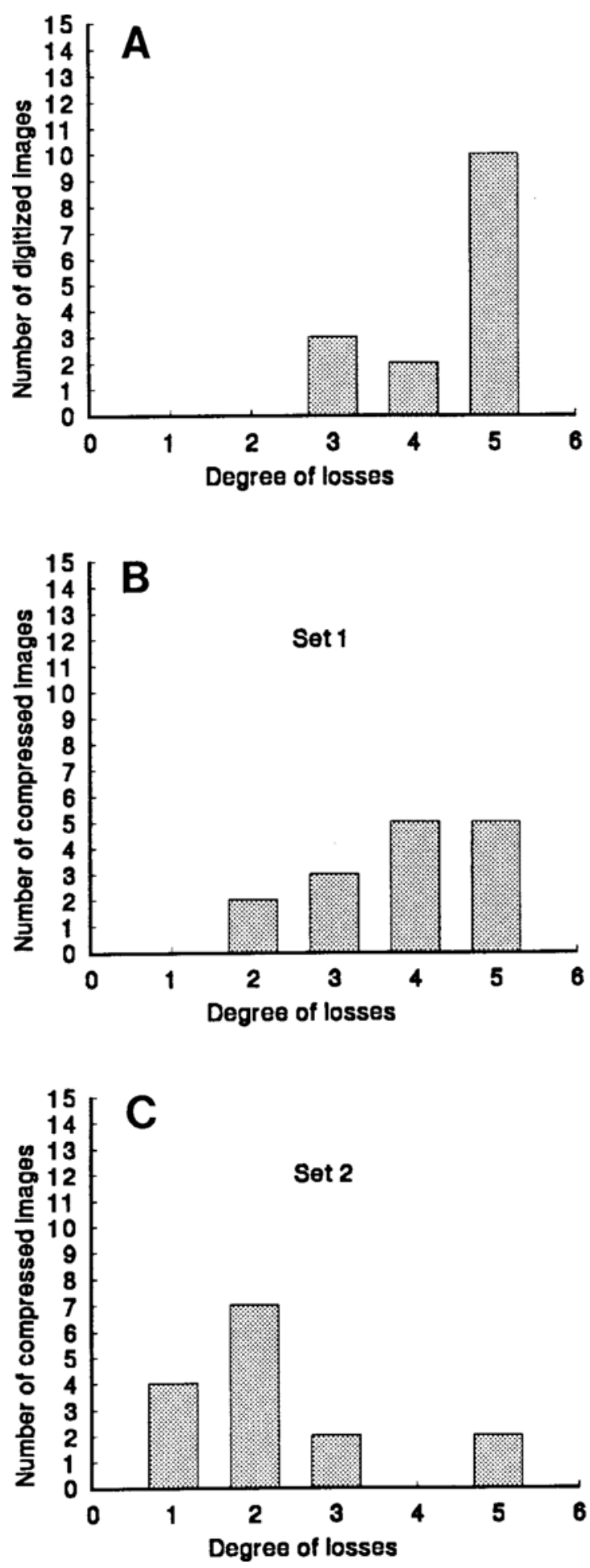

Fig 8. Bar diagrams of the grading of the overall losses in (A) the uncompressed digitized images, (B) the first set of compressed images, and $(C)$ the second set of compressed images. The scale is 1 , extreme; 2 , high; 3 , average; 4 , low; 5 , none. 
pression data have similar morphology as the digitized mammograms and the corresponding films; the remaining $47 \%$, the majority of which is from the 10-bit images, shows a one-level degradation in the morphology of the calcifications. This also explains the differences in the suspiciousness of the cluster. (5) The digitized mammograms reproduce exactly the parenchyma patterns observed on the film. In $33 \%$ of the cases, the first compression set did not show any visible distortion of the parenchymal tissues (the majority of these cases were from the group of the 12-bit images). In all other cases the smoothness and continuity of the parenchymal tissues is gradually lost and the compressed data have a "boxy" appearance which increases with increasing compression ratio.

\section{CONCLUSIONS}

We have applied Haar wavelets for the compression of mammographic images with calcification clusters aiming at the highest possible compression with clinically acceptable lossy data. Two high compression rates were tested to determine the degree of losses and their effect on the visualization of malignant calcifications. The Haar wavelets introduced losses in the smoothness and continuity of the pixels in the original data but preserved the calcifications as indicated by the differences between the original and compressed images and the fact that the visualization of the calcifications was rated as excellent by the radiologist for compressions up to 56:1. Furthermore, the expert mammographer could not discriminate the digitized from the decoded mammogram in $80 \%$ of the cases. However, the results seem to depend on the contents of the original image and the dynamic range of the digital mammogram. It also appears that decoded images with higher dynamic ranges were evaluated more favorably despite the fact that the display of the images is limited by the current technology to 8 bits per pixel. Further studies are needed if a digitization strategy more beneficial to the compression process is to be determined.

A wavelet segmentation algorithm was also applied to both uncompressed and compressed data to determine whether it could partially compensate for the compression losses. The results showed that this algorithm, at its present design, successfully segments the images but generates several false-positive signals in the highly compressed data. Motivated by the results of the present study, we are now engaged in the implementation of smoother wavelet approaches for the compression of mammograms and the improvement of the wavelet segmentation algorithm to reduce the falsepositive detection rate which may, in turn, modify the clinically acceptable limits of lossy compression. A more extensive application of wavelets to digital mammography including clinical evaluation and confirmation of our preliminary results is ongoing.

\section{REFERENCES}

1. Chan HP, Vyborny CJ, MacMahon J, et al: Digital mammography ROC studies of the effects of pixel size and unsharp-mask filtering on the detection of subtle calcifications. Invest Radiol 22:581-589, 1987

2. Davies DH, Dance DR: The automatic detection of subtle calcifications in high resolution, high quality mammograms. Phys Med Biol 37:1385-1390, 1992

3. Bassett LW, Gambhir S: Breast imaging for the 1990s. Semin Oncol 18:80-86, 1991

4. Wilson DL: Compression for radiological images. Proc SPIE Conference on Medical Imaging VI: PACS Design and Evaluation 1654:130-139, 1992

5. Kuduvalli GR, Rangayyan RM: Performance analysis of reversible image compression techniques for highresolution digital teleradiology. IEEE Trans Med Imag 11:430-445, 1992

6. Lelewer D, Hirshberg D: Data compression. ACM Computing Surveys 19:261-296, 1991

7. Richardson WB: Wavelets packets applied to mammo- grams. Proc IS\&T/SPIE Annual Symposium on Electronic Imaging, Science \& Technology, San Jose, CA; January 31-February 5, 1993

8. Magnin IE, Baudin O, Baskurt A, et al: An adaptive coding algorithm for a mammogram image database. Proc IS\&T/SPIE Annual Symposium on Electronic Imaging, Science \& Technology, San Jose, CA; January 31-February 5,1993

9. MacMahon H, Doi K, Sanada S, et al: Data compression: Effect of diagnostic accuracy in digital chest radiography. Radiology 178:175-179, 1991

10. Chen J, Flynn MJ: The effect of block size on image quality for compressed chest radiographs. Proc SPIE Conference on Image Capture, Formatting and Display, 1653:252260,1992

11. DeVore R, Jawerth B, Lucier B: Image compression through wavelet transform coding. IEEE Trans Inf Theory 38:719-746, 1992

12. Qian W, Clarke LP, Kallergi M, et al: Tree- 
structured nonlinear filter and wavelet transform for microcalcification segmentation in mammography. Proc IS\&T/ SPIE Annual Symposium on Electronic Imaging, Science \& Technology, San Jose, CA; January 31-February 5, 1993

13. Clarke LP, Kallergi M, Qian W, et al: Treestructured filter and wavelet transform for microcalcification segmentation in digital mammography. Cancer Letters, 1994 (in press)

14. Qian W, Clarke LP, Kallergi M, et al: Tree structured nonlinear filters in digital mammography. IEEE Trans Med Imag, 1993 (submitted)

15. Mallat S: A theory for multiresolution signal decomposition: The wavelet representation. IEEE Trans Patt Anal Machine Intel 11:674-693, 1989

16. Mallat S: Multifrequency channel decompositions of images and wavelet models. IEEE Trans Acoust Speech Signal Proc 37:2091-2110, 1989 